\title{
Anomalous foreshock field-aligned beams observed by Cluster
}

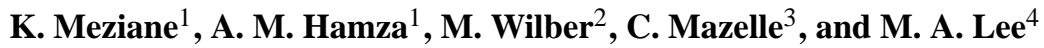 \\ ${ }^{1}$ Physics Department, University of New Brunswick, Fredericton, Canada \\ ${ }^{2}$ Space Sciences Laboratory, University of California, Berkeley, USA \\ ${ }^{3}$ Centre d'Etude Spatiale des Rayonnements, Toulouse, France \\ ${ }^{4}$ Space Science Center, University of New Hampshire, Durham, USA
}

Received: 29 June 2011 - Revised: 4 October 2011 - Accepted: 24 October 2011 - Published: 31 October 2011

\begin{abstract}
We report occasional observations of two simultaneously distinct ion foreshock components recorded by the Cluster spacecraft upstream of the Earth's bow shock. In most occurrences, the lower-energy population originates as a field-aligned beam (FAB) associated with quasiperpendicular regions, which loses energy as the IMF rotates into oblique geometries. A second beam, with energies in excess of $\sim 10 \mathrm{keV}$, appears sometimes in association with the onset of ultra-low frequency (ULF) waves, and sometimes ahead of the appearance of the latter. Measurements from the mass spectrometer indicate that both beams consist of protons. While the lower-speed beam is wellaccounted for by a known reflection mechanism, the nonradial IMF orientations as well as other arguments seem to rule out magnetosheath or magnetospheric sources for the higher energy component. The wave characteristics are typical of the oblique foreshock and we have found that they are in cyclotron-resonance with the low speed beam (FAB). These observations constitute a theoretical challenge since conventional mechanisms described in the literature cannot account for the production of beams at two different energies.
\end{abstract}

Keywords. Interplanetary physics (Interplanetary magnetic fields; Planetary bow shocks; Solar wind plasma)

\section{Introduction}

It is well recognized that the characteristics of regions upstream of collisionless shocks are strongly controlled by shock geometry, which is determined by the angle $\theta_{B n}$ between the upstream magnetic field and the shock normal direction. Field-Aligned Beams (FABs) are a prominent fea-

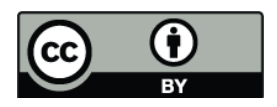

Correspondence to: K. Meziane (karim@unb.ca) ture of the terrestrial foreshock region for $40^{\circ} \lesssim \theta_{B n} \lesssim 70^{\circ}$. They consist of ion populations propagating sunward away from Earth's bow shock, collimated along the interplanetary magnetic field (IMF) while subject to the $\boldsymbol{E} \times \boldsymbol{B}$ drift. In a time-energy spectrogram, they appear as narrow energy bands significantly above the solar wind spectrum. Their source regions and mechanims have been studied extensively, in part because their streaming motion relative to the solar wind is an important source of free energy. ULF waves are common in oblique $\left(25^{\circ} \lesssim \theta_{B n} \lesssim 50^{\circ}\right)$ and quasi-parallel $\left(\theta_{B n} \lesssim 35^{\circ}\right)$ foreshock regions, but are absent where FABs are observed. However, it is common for FABs to have associated whistler-like waves, with typical frequencies $\sim 1 \mathrm{~Hz}$.

Despite significant progress, a full understanding of their production mechanism remains elusive. However, recent studies have considered two mechanisms that could account for FAB production. A leading idea stipulates that they originate from specularly reflected gyrating ion distributions produced at the quasi-perpendicular shock ramp (Möbius et al., 2001; Kucharek et al., 2004). Since specular reflection of the solar wind in quasi-perpendicular geometries results in streaming motions directed toward the magnetosheath, vigorous pitch-angle scattering is required to redirect a significant number of ions sunward along the IMF direction. Such a scattering mechanism, which should operates on very short time scales, has yet to be identified. Moreover, there should result from this mechanism noticeable quantitative changes in FAB distribution functions as $\theta_{B n}$ varies. In another production mechanism, FABs are produced by shock drift acceleration, in which the particle energization results from gradient drift motions along the shock surface parallel to the tangential component of the motional electric field (Paschmann et al., 1980). Where this mechanism satisfactorily accounts for the beam bulk speed, it suffers from the injection problem, as only a tiny portion from the tail of the incident solar wind beam distribution has pitch angles that should result in mirroring in the shock ramp (Meziane et al., 2005).

Published by Copernicus Publications on behalf of the European Geosciences Union. 


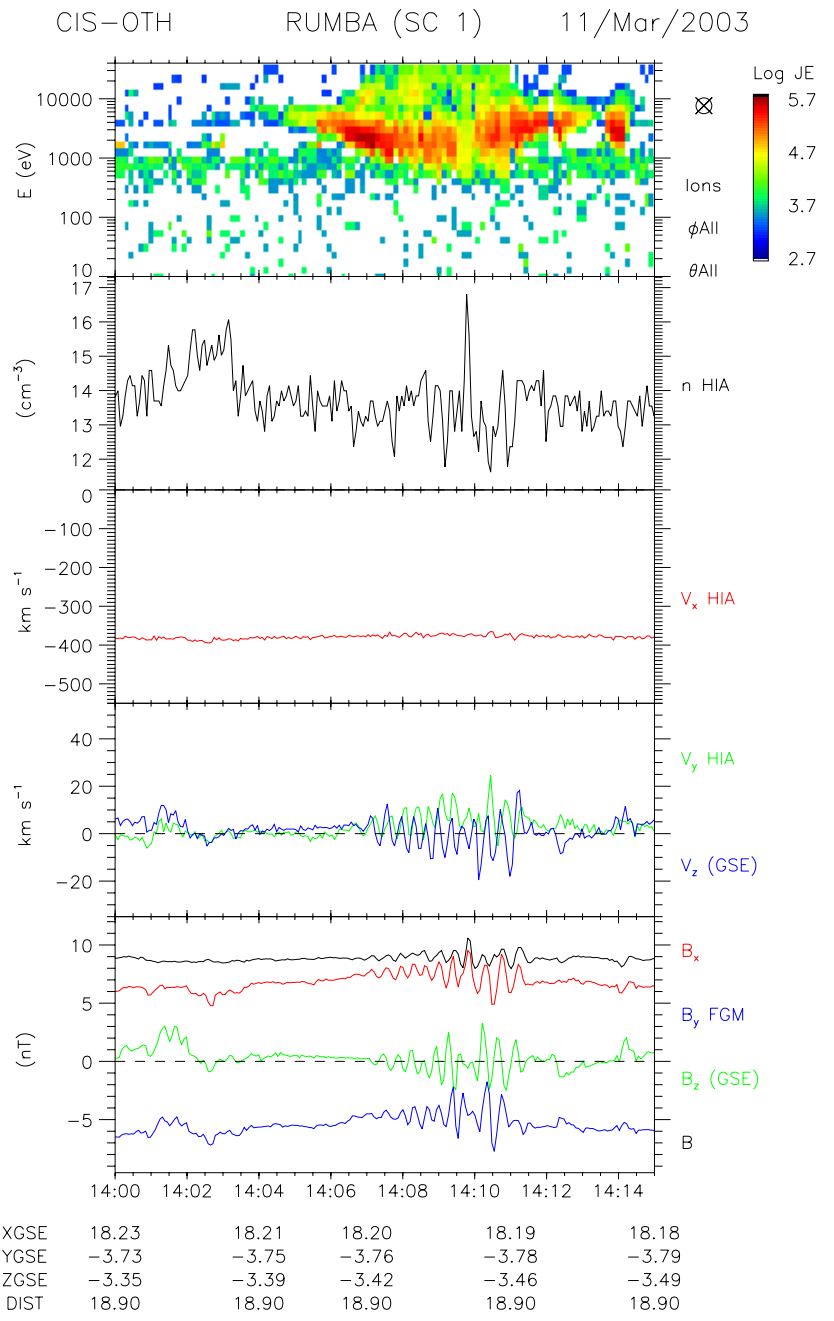

Fig. 1. Top panel shows the time-energy spectrogram from the CISHIA sensor for 11 March 2003, 14:00-14:15 UT. The successive panels display respectively the ion density, the GSE components of the solar wind velocity, the interplanetary magnetic field GSE components and magnitude from FGM measurements.

A detailed analysis of FAB velocity distributions reveals that most of their ions emanate from a narrowly-restricted source region just upstream of the point where the IMF threads the spacecraft. In fact, a recent investigation has revealed that some ion trajectories, with shock footpoints and the potential to reach the spacecraft, do not make it to the spacecraft (Meziane et al., 2010).

The different types of foreshock ion populations, FABs, intermediate/gyrating ions, and diffuse ions, are spatially well segregated. One of the very few counter-examples in the literature was a case where gyrating ions were seen coincident with FABs. Taking into consideration the observed gyrophases of the gyrating population, it became clear that these were seen because their guiding centers were within a gyroradius of the FABs, leading to some overlap (Meziane et al., 2004b). In another study, Kis et al. (2007) reported a FAB distribution with a significant high energy tail. The authors argued that the tail is associated with ions emanating from the quasi-parallel shock region while it is known that FABs are seen on IMF lines connected to quasiperpendicular geometries. However, this possibilty may be observed when the spacecraft is located near a boundary between the FAB and the diffuse ion populations The results presented below are of particular interest, because we report for the first time the occurrence of distinct, simultaneous field-aligned beam populations. A simple analysis indicates that the occurrence of such a secondary beam does not fit the conventional quasi-perpendicular foreshock topology.

After brief description of the instrumentation onboard of the spacecraft, we detail in the next section Cluster observations of simultaneously occurring FABs. In Sect. 3, we characterize the populations according to possible source mechanisms, and follow in Sect. 4 with a discussion of the obtained results.

\section{Observations}

The particle data used in the present study are from the Cluster Ion Spectrometer (CIS) experiment, which includes a top-hat electrostatic analyzer (HIA) and a mass spectrometer (CODIF). The velocity distributions are obtained from the HIA instrument, which accumulates full three-dimensional (3-D) distributions functions within one spin period (4s), with an angular resolution of $22.5^{\circ} \times 22.5^{\circ}$. The HIA sensor spans an energy range of $0.005-38 \mathrm{keV} \mathrm{q}^{-1}$. In normal telemetry mode, however, one distribution is transmitted every 2 or 3 spins depending on the time period, whereas in burst mode, an HIA distribution is transmitted every spin. An extensive description of the CIS experiment is found in (Rème et al., 2001). Our study also uses magnetic field data from the fluxgate magnetometer (FGM) (Balogh et al., 2001). We now present the observation sample consisting of three events which illustrate our finding; only the first event will be examined in detail.

\subsection{Event-1: 11 March 2003, 14:00-14:15 UT}

The top panel of Fig. 1 shows the time-ion energy spectrogram measured by Cluster 1-HIA on 11 March 2003, for the time interval 14:00-14:15 UT. The Cluster 1 spacecraft location was $(18.20,-3.76,-3.42) R_{\mathrm{E}}$ from Earth. The subsequent panels show the onboard plasma density, velocity components, and the three GSE-components of the interplanetary magnetic field with 4s-resolution, respectively. When in solar wind mode, the HIA high geometry factor analyzer truncates the energy sweep above solar wind energies when looking sunward, in order to minimize saturation damage. Consequently, the energy fluxes shown in the timeenergy spectrogram corresponds almost exclusively to backstreaming ions; the remnant of the solar wind spectrum is 

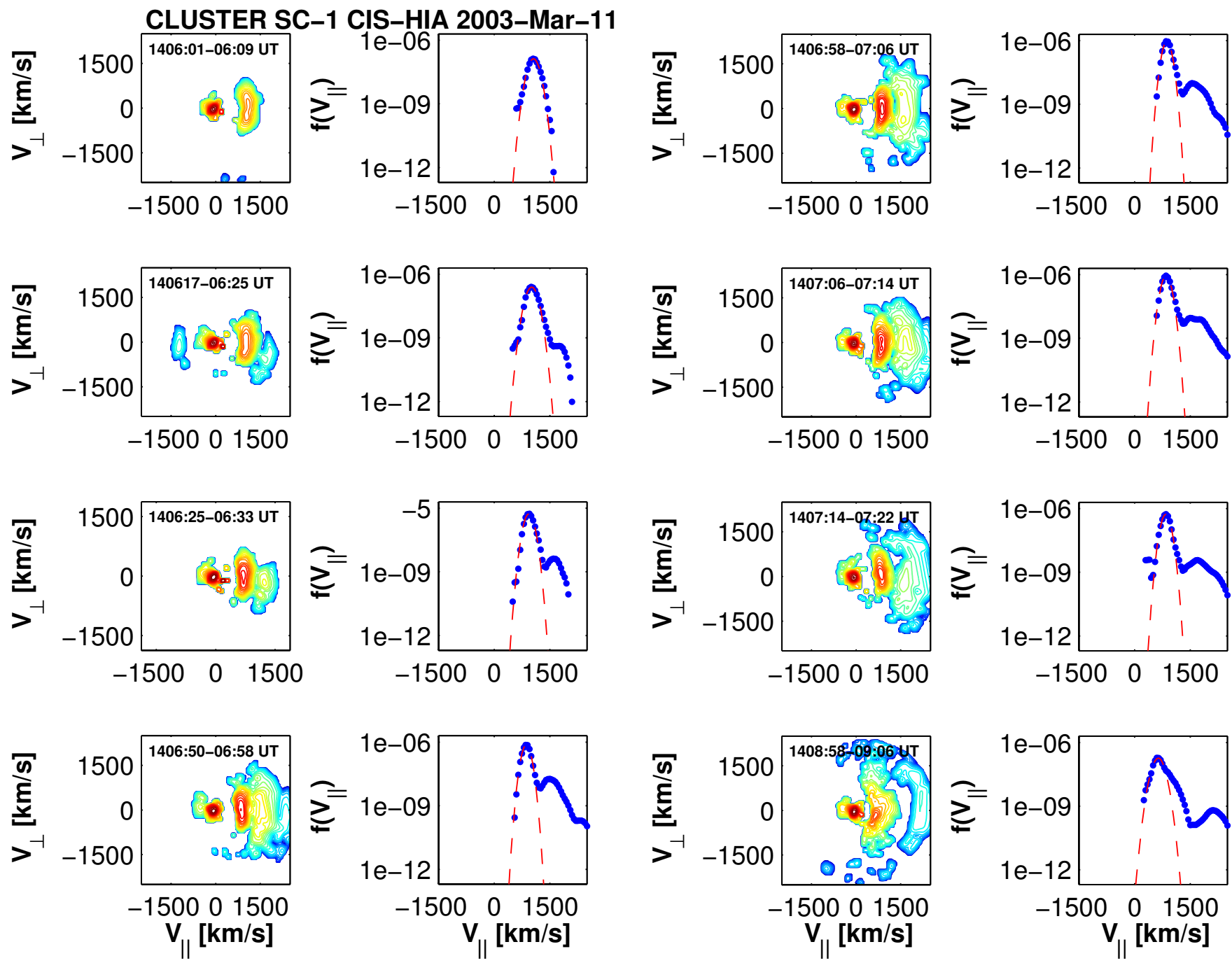

Fig. 2. Successive left panels show the ion velocity distribution in the $v_{\|}-v_{\perp}$ system of coordinates and in the plasma frame of reference; the contours at the origin are associated with the solar wind measurement. Successive right columns show the corresponding reduced parallel distribution functions $f\left(v_{\|}\right)$; the solar wind has been removed. The dashed red line represents the best Gaussian fit of the FAB distribution component. The contour levels of the space phase density are coded such that the deep red corresponds to $10^{-6} \mathrm{~cm}^{-3} /\left(\mathrm{km} \mathrm{s}^{-1}\right)^{3}$ and the purple to $10^{-14} \mathrm{~cm}^{-3} /\left(\mathrm{km} \mathrm{s}^{-1}\right)^{3}$.

indicated by the green narrow band just below $\sim 1 \mathrm{keV} \mathrm{q}^{-1}$. From 14:04:00 UT, as a magnetic connection to the shock occurs, a FAB ion population appears with an increasing flux and decreasing in bulk energy. This noticeable variation is due to the monotonic rotation of the y-component of the IMF. The FABs persist until 14:08:00 UT when the spectra are wider, and at the same time significant coherent fluctuations appear in the magnetic field data. For as long as FABs are present, very weak or no magnetic fluctuations are noticed. This description depicts perfectly the conventional picture of quasi-perpendicular foreshock regions. On top of this common foreshock feature, an additional population $\left(E \geq 10 \mathrm{keV} \mathrm{q}^{-1}\right)$, is seen to branch off from the moreintense FABs just before 14:06:15 UT, and persists for few minutes. We emphasize that this additional ion component is visible about a half minute before the appearance of prominent coherent magnetic field fluctuations near $\sim 14: 07$ UT. We next examine the velocity distributions obtained during this time interval.

We present in Fig. 2 successive ion distribution functions, which illustrate the situation depicted above. Panels in the first and third columns show the full 3-D distribution functions folded onto the $v_{\|}-v_{\perp}$ plane; the velocities are sampled in the plasma frame of reference. Panels in the second and fourth columns show reduced distributions integrated over $v_{\perp}$ corresponding to the full distributions to their left. The contours corresponding to the solar wind remnant have been eliminated in the reduced parallel distribution functions. The 


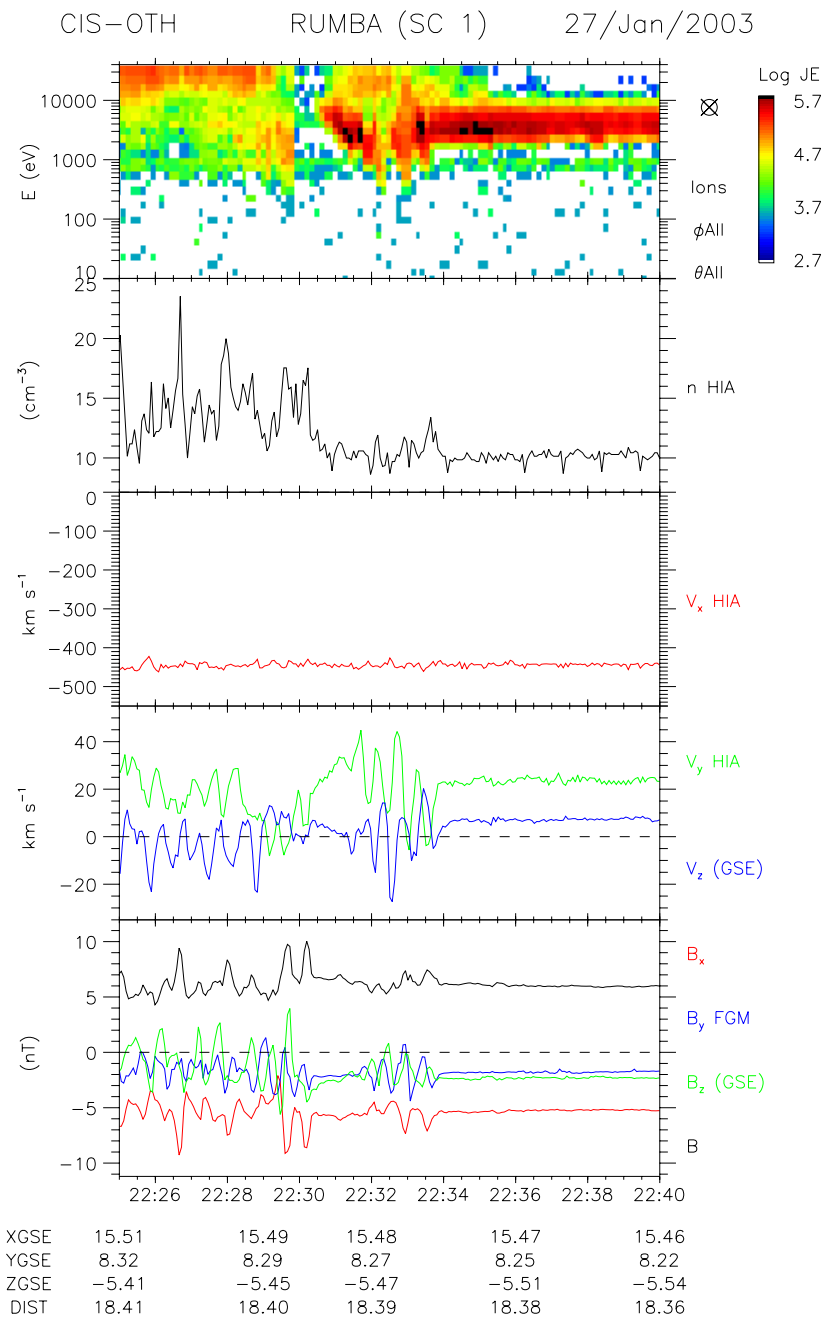

Fig. 3. Cluster-CIS and FGM observations for 27 January 2003. The format is the same as Fig. 1.

top left panels clearly indicate a signature of a FAB population, with a bulk speed on the order of $\sim 1060 \mathrm{~km} \mathrm{~s}^{-1}$. The FAB population persists for the subsequent snapshots, but is accompanied by an additional population which progressively appears to the right of the FAB at a higher bulk speeds. The additional population is clearly distinguished in many snapshots, with the bulk motion seemingly collimated along the IMF direction, although a small departure from the latter direction is not ruled out.

As a first attempt to explain the atypical ion distribution, we considered that the secondary beam might consist of a different species, such as $\mathrm{He}^{++}$, the most abundant atomic mass number $A>1$ present in the solar wind. We examined the CODIF spectra and time of flight diagnostics during the time of interest and found that these two distinct populations are associated with protons. Moreover, we found no evidence for the presence of any other heavy ions such as $\mathrm{He}^{+}$or $\mathrm{O}^{+}$. To our knowledge, such a signature of distinct,

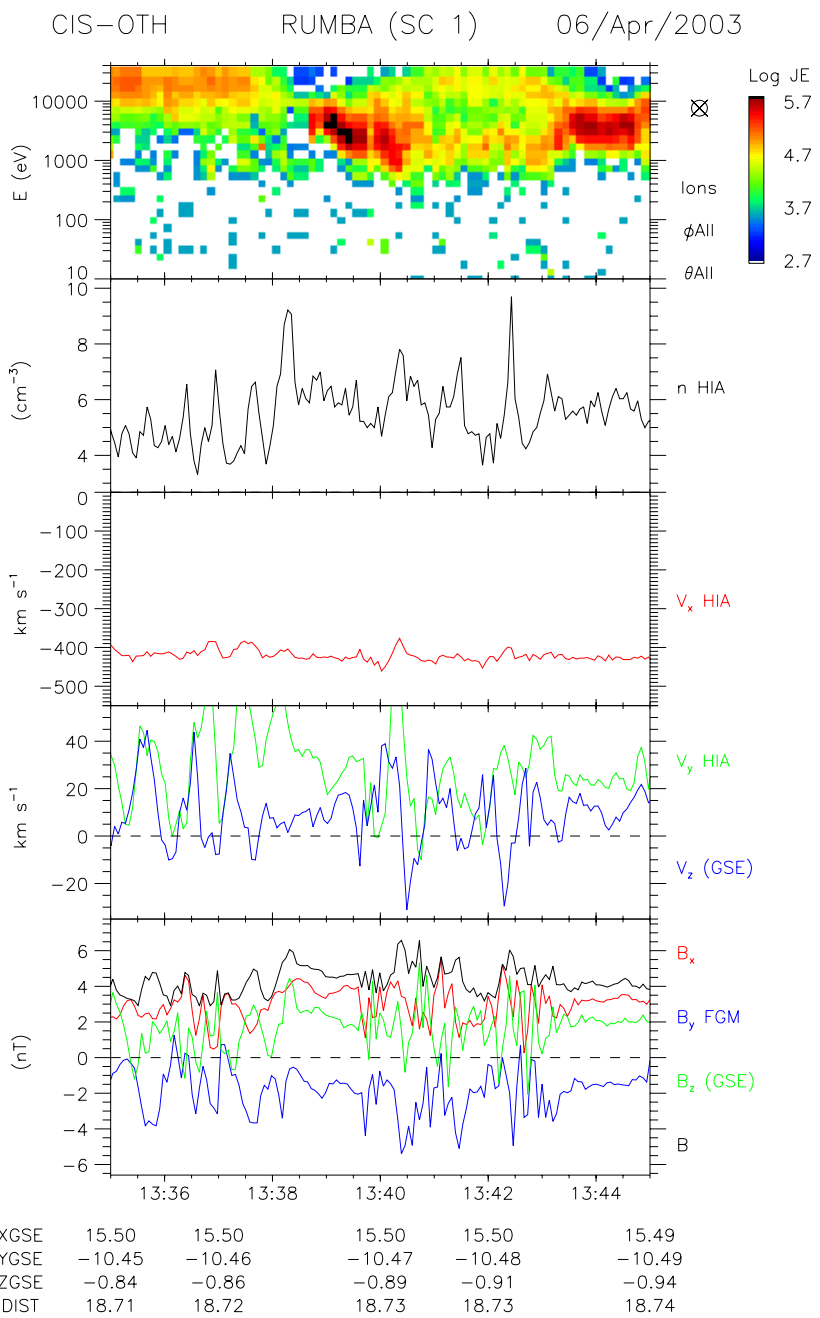

Fig. 4. Cluster-CIS and FGM observations for 6 April 2003. The format is the same as Fig. 1.

energetic backstreaming, field-aligned proton beams has not been reported before.

\subsection{Event-2: 27 January 2003, 22:25-22:40 UT}

Figure 3 shows another Cluster CIS/HIA event of interest recorded on the 27 January 2003, 22:25-22:40 UT. The format of Fig. 3 is the same as Fig. 1. The onset of FABs at 22:30:30 UT indicates an encounter with the foreshock, and recent magnetic connection with the shock. The decrease in beam speed corresponds to a simultaneous, monotonic IMF rotation. A distinct beam with a higher $\left(>10 \mathrm{keV} \mathrm{q}^{-1}\right)$ energy appears at $\sim 22: 31: 15 \mathrm{UT}$, and persists for about one minute. As we noted previously, the secondary beam branches from the main, lower-energy FAB, before the ULF wave packet attains maximum amplitude. While the rotation of the IMF leads to an expected decrease in energy for the main beam, the secondary beam initially gain energy and thereafter maintain the $20-30 \mathrm{keV} \mathrm{q}^{-1}$ peak levels. 


\subsection{Event-3: 6 April 2003, 13:35-13:45 UT}

Figure 4 shows observations from the 6 April 2003, which are presented using the same format as Fig. 1. Prior to 13:38 UT we observe strong ULF waves in the bottom panel, which exhibit significant compressional power in addition to strong transverse wave power. A sharp increase in the solar wind density at this time from $5-8 \mathrm{~cm}^{-3}$ pushed the shock inwards, leading to a momentary disconnection at the spacecraft. At $\sim 13: 41: 30 \mathrm{UT}$, the ion spectrum is split into two relatively narrow components; the beams merge at $\sim 13: 42: 30$ UT into a typical FAB population. Although this event qualitatively contrasts with the first two, it also illustrates a case of a simultaneous observations of two distinct foreshock populations merging into FABs.

\section{Characterization}

Given the quality of the data, a quantitative characterization of both beams is certainly of interest for the study. For this purpose, only the 11 March 2003 event will be considered here. First, we determine the bulk parallel speed associated with each beam. For a precise determination, we used a Maxwellian to fit the phase space density peak of the reduced distributions. Normalized to the solar wind speed $\left(v_{\mathrm{SW}} \sim 380 \mathrm{~km} \mathrm{~s}^{-1}\right)$, the resulting numerical values for the speed of the main beam $\left(P_{\mathrm{FAB}}\right)$ and the secondary beam $\left(p_{2}\right)$ are listed in columns 2 and 3 of Table 1 for successive events, respectively.

We note that the secondary beam is not present for the first two snapshots. We also notice that the FAB beam speed continuously decreases, a change consistent with the slow monotonic IMF rotation (y-component) leading to changes in shock geometry. On the other hand, the secondary beam speed exceeds that of the FAB by several times its thermal width. It also seems that its changes are not controlled by the IMF rotation. Pursuing our analysis, we provide an estimation of the shock geometry associated with the events as well the possible ion shock source region. For this purpose, and for each event listed in Table 1, we map the guiding centre path between the spacecraft location and the bow shock. For analytical simplicity, we assume a stationary upstream solar wind and IMF conditions, and the field lines are assumed to be straight. For zero pitch angle ions, the guiding centre orbit lies in the $B v_{\mathrm{SW}}$-plane (containing both the solar wind velocity and IMF directions). FAB ions reaching the spacecraft all emanate from a region where the $B v_{\mathrm{SW}}$-plane intercepts the shock. The shock is modelled as a paraboloid shape with nominal values for the sub-solar distance $a_{\mathrm{S}}$ and the flaring parameter $b_{\mathrm{S}}=\frac{1}{4 L}$, where $L$ is the semi-latus rectum. We used Cairns et al. (1995) to define the model, and based on solar wind parameters measured at the time of interest, we obtained $a_{\mathrm{S}}=12.1 R_{\mathrm{E}}$ and $b_{\mathrm{S}}=0.0252 R_{\mathrm{E}}^{-1}$. Using the bulk speeds of both beams, we then determined the in- tercept with the bow shock of each guiding centre path as well as the intersection of the IMF line threading the spacecraft with the shock. We emphasize that all the analytical computations are derived in 3-dimension. Columns 5-7 of Table 1 report the the GSE-coordinates of the position where the secondary beam path intersects the shock with respect to the main beam (FAB). Finally, we have estimated the angles $\theta_{V n}$ and $\theta_{B n}$ that the shock normal makes with the solar wind and IMF directions, respectively. Both angles are calculated at the shock location where the IMF line threading the spacecraft intersects the shock, as well as at the ion source, when the solar wind convection is taken into account. In the plasma rest frame of reference, the shock speed $v_{\text {shock }}$ is given by the following expression:

$P_{\text {shock }}=\frac{v_{\text {shock }}}{v_{\text {SW }}}=-\frac{\cos \theta_{V n}}{\cos \theta_{B n}}$

In Eq. (1), $\theta_{V n}$ is obtuse whereas $\theta_{B n}$ is acute. As mentioned in Sect. 1, FAB speeds are usually consistent with the prediction of a quasi-adiabatic reflection of a portion of the incoming solar wind ions. The mechanism predicts a post encounter normalized parallel speed given by (Meziane et al., 2010):

$p=\frac{v_{\|}}{v_{\mathrm{SW}}}=-(1+\delta) P_{\text {shock }}$

where $0<\delta \leq 1$. The reflection is adiabatic for $\delta$ equal to unity. Previous FAB observations indicated that $\delta$ is in the range 0.85-1.0 (Bonifazi et al., 1983; Paschmann et al., 1980).

Rigorously, the angles in Eq. (1) should be determined at the source point (the point where the guiding centre path threading the spacecraft intercepts the shock). Their determination implicitly assumes that the IMF is uniform at least for the time it takes for an ion to travel from the shock to the spacecraft. However, in the literature, the shock angles are usually calculated at the point where the IMF line threading the spacecraft intercepts the shock (Paschmann et al., 1980; Schwartz et al., 1983; Thomsen et al., 1983), and have been used for comparison with theoretical models. We calculated the shock speed and deduced the theoretical FAB speed for each distribution of Fig. 2. From Table 1, it is clear that the FAB beam speed consistently satisfies Eq. (2). In the case of the last distribution at 14:08:58-09:06 UT, the agreement with Eq. (2) is violated; a detailed examination indicates that the distribution corresponds a to gyrating ion population rather than a FAB. When the shock geometry is determined at the point where the IMF line threading the spacecraft is considered, as in previous studies, Table 1 indicates that $\delta \sim 1$ and remains constant; while when the solar wind convection is taken into account, there is a loss of momentum $(\delta \sim 0.7)$. This agreement with the reflection model, as well with previous studies, indicates that the primary beam agrees very well with the known characteristics of foreshock FABs. On the contrary, and as indicated by column 3 of Table 1 , the secondary beam speed is significantly larger than what could be 
Table 1. Beam speeds and bow shock connection for a paraboloid shock model.

\begin{tabular}{lcccccc}
\hline $\begin{array}{l}\text { Snapshot } \\
\text { (UT) }\end{array}$ & $P_{\mathrm{FAB}}$ & $p_{2}$ & $P_{\text {shock }}$ & $\begin{array}{c}\Delta X_{2-1} \\
\left(R_{\mathrm{E}}\right)\end{array}$ & $\begin{array}{c}\Delta Y_{2-1} \\
\left(R_{\mathrm{E}}\right)\end{array}$ & $\begin{array}{c}\Delta Z_{2-1} \\
\left(R_{\mathrm{E}}\right)\end{array}$ \\
\hline 14:06:01-06:09 & 2.79 & - & 1.31 & - & - & - \\
14:06:17-17:25 & 2.66 & - & 1.27 & - & - & - \\
14:06:25-06:33 & 2.47 & 4.31 & 1.23 & 0.737 & -3.932 & -0.194 \\
14:06:50-06:58 & 2.28 & 3.90 & 1.18 & 0.593 & -2.699 & -0.145 \\
14:06:58-07:06 & 2.29 & 4.08 & 1.14 & 0.376 & -2.176 & -0.112 \\
14:07:06-06:14 & 2.24 & 3.90 & 1.14 & 0.418 & -2.384 & 0.042 \\
14:07:14-07:22 & 2.22 & 4.47 & 1.15 & 0.528 & -2.918 & 0.116 \\
14:08:58-09:08 & 1.68 & 5.84 & 1.17 & - & - & - \\
\hline
\end{tabular}

Table 2. Properties of the waves.

\begin{tabular}{lccccccc}
\hline Time interval & $\lambda_{2} / \lambda_{3}$ & $\lambda_{1} / \lambda_{2}$ & $T_{C}$ & $T$ & $\theta_{k V}$ & $\theta_{k B}$ & $P_{\text {res }}$ \\
\hline 14:07:02-07:46 UT & 40 & 1.4 & $7.4 \mathrm{~s}$ & $24 \mathrm{~s}$ & $56^{\circ}$ & $27^{\circ}$ & $2.04 \pm 0.22$ \\
\hline
\end{tabular}

expected from a simple adiabatic reflection. Finally, we have checked that the shock geometry determination based upon the use of parabolic bow shock is very similar to that obtained from an hyperboloid model (Slavin and Holzer, 1981) or an elliptical model (Farris et al., 1991).

The bottom panel of Fig. 1 displays significant amplitude fluctuations of the DC magnetic field components, which appear after $\sim 14: 06: 30 \mathrm{UT}$ and last a few cycles. In order to quantify a possible ULF waves link with the beams, we carried out the standard wave analysis on a selected time interval of interest. We applied the standard minimum variance analysis (MVA) technique (Sonnerup and Scheible, 1998) to fully characterize the observed waves. The usual convention is used to order the eigenvalues of the covariance matrix of the field perturbations $\left(\lambda_{1}>\lambda_{2}>\lambda_{3}\right)$ (maximum, intermediate and minimum variances, respectively). The direction of minimum variance gives the direction of propagation with respect to the ambient field $\boldsymbol{B}_{0}$ computed as the averaged field vector during the time interval analyzed. The wave properties obtained, as summarized on Table 2, depicts a case of typical ULF waves seen at oblique shocks: the waves are nearly circular $\left(\lambda_{1} / \lambda_{2} \sim 1.4\right)$ and left-hand polarized with respect to the ambient magnetic field in the spacecraft frame of reference. The results indicate that the wave field vector remains in a well-defined plane while the direction of propagation is determined with a satisfactory precision. The analysis of an adjacent time interval leads to similar results, which suggests that the results obtained are experimentally reliable. The quasi-monochromatic nature of the observed waves strongly suggests a local production. Although the MVA technique is degenerate with respect to the signs of the eigenvectors, numerous prior studies indicate that these waves propagate upstream, and a corresponding selection of sign has been adopted.
The energy source and the ULF wave activity have been thoroughly investigated in past studies (Gary et al., 1981; Meziane et al., 2001). It is not the goal of the present study to provide a detailed investigation of the wave-particle interactions. However, based upon convincing studies, the commonly accepted explanation for the wave generation stipulates that the ULF growth is attributed to nonlinear beam disruption (Mazelle et al., 2000, 2003). In this case, the cyclotron resonance with beams might be considered a strong indication for local wave production. Therefore, it is instructive to estimate the cyclotron resonance speed in the present case. In a low frequency regime, $\omega<\Omega_{i}$ and $\omega_{\mathrm{sc}}>>\omega$, where $\omega, \omega_{\mathrm{sc}}$, and $\Omega_{i}$ are the wave frequencies in the plasma and spacecraft frames and the ion gyrofrequency, respectively. It is therefore straightforward to show that the cyclotron resonant speed (normalized to the solar wind speed) is given by:

$P_{\text {res }}=\frac{\Omega_{i}}{\omega_{\text {sc }}} \frac{\cos \theta_{k V}}{\cos \theta_{k B}}$

In Eq. (3), $\theta_{k B}$ and $\theta_{k V}$ correspond to the angle that the direction of wave propagation makes with the ambient field and the solar wind directions, respectively. The numerical value, $P_{\text {res }}=2.04 \pm 0.22$, provided in the last column of Table 2, strongly supports the possibility of a local cyclotron resonance with the FABs; particularly those FABs observed at the onset of the wave trains. This is much lower, however, than those obtained for the secondary $p_{2}=3.90-5.85$ (column 3 of Table 1), so we can rule out any resonance in their case. 


\section{Discussion}

In this brief report, we have presented Cluster-CIS observations of anomalous foreshock beams. Observed simultaneously with ordinary FABs, these high energy beams propagate upstream away from the shock with speeds three to four time the shock speed. This secondary field-aligned population first appears nearly coincident with the onset of ULF waves, but well before they reach high amplitudes. But as we discuss below, these do not fit within present foreshock beam production paradigms.

We considered the possibility that the secondary beam consists of an ion species different from protons, and examined data from the CIS/CODIF ion composition instrument on Clusters 1 and 4. The instrument reveals that all the nonthermal ions consist of protons. Also, we found no evidence for magnetospheric tracers such as $\mathrm{O}^{+}$. Moreover, we could estimate the energy/charge expected for solar origin species other than protons, assuming that they travel at the solar wind speed and also specularly reflect, as $\left(e / q_{\mathrm{i}}\right)\left(m_{\mathrm{i}}^{2} / m_{p}^{2}\right)$ times that for protons. The measured values for the secondary beam peak are much larger than double that for the main $\mathrm{FAB}$, as would be expected for $\mathrm{He}^{++}$.

The simultaneous observation of two foreshock populations might result as a direct remote sensing effect, as in the picture presented in (Meziane et al., 2004b). Also, the absence of a dependence of the higher-energy beam population on the IMF direction potentially could be consistent with a source based in diffuse populations associated with oblique and quasi-parallel shock geometries. In that case, the secondary beam ions would have guiding centers located on field lines downstream of and adjacent to those of the FABs. We believe that this possibility is not a suitable explanation. First, the high energy beam is highly field-aligned, ruling out a remote sensing effect. Also, the $E>7 \mathrm{keV}$ angular distributions for the 11 March 2003 event (not shown) provide no evidence that ions are restricted in gyrophase, as should be the case for remote sensing. Previously, Oka et al. (2005) and Meziane et al. (2007) reported high-energy tails on some FAB distributions, with the tails being associated with energetic gyrating ions. Although investigated in detail using Cluster-CIS data, the production of these high-energy tails remains unresolved (Meziane et al., 2007). While some distributions in Fig. 2 allow for the possibility of small, non-zero pitch angles for the secondary beams, the absence of a gyrophase dependence makes gyrating ions an unlikely source. Oka et al. (2005) suggested that the gyrating component may result from a finite Larmor radius effect, but did not investigate that possibility further. While the present observations of high-energy beams suggest a relationship to the occurrence of ULF waves, no such waves were observed in association with FABs having nongyrotropic high energy tails (Meziane et al., 2007). This does not support an obvious link between the results we report here and those FABs with high-energy tails. Moreover, the energetic component could not be accounted for by the mechanism due to Möbius et al. (2001) - a specular reflection followed by pitch-angle scattering in the shock frame. Were we to suppose that both beams were produced in this manner, the difference would be that the energetic component is supposed to be produced at more quasi-parallel geometries, where the shock speed and hence the reflection speed are lower. Assuming instead that the primary FAB population is produced by a mirroring process, we have found it to be consistent with a nearly energyconserving case $(\delta \sim 1)$, for which, again, the beam speeds should be higher than those for reflected particle produced at lower values of $\theta_{B n}$.

The possibility that magnetosheath leakage could be a source for the secondary also is not supported by the data. Magnetosheath upstream escape models based on the conservation of the magnetic moment $\mu=v_{\perp}^{2} / B$ (Edmiston et al., 1982; Schwartz et al., 1983; Meziane et al., 2004b) predict field-aligned distributions that are peaked at the shock speed, and therefore do not account for the high speed beam production. The possibility that the secondary beam might result from resonant wave-particle processes is not supported by the wave analysis. In agreement with previous studies, the wave properties strongly indicate a resonant interaction between the waves and the ordinary FAB ions, resulting in beam disruption when the wave amplitude is substantial.

The beam shape of the anomalous population might indicate a coherent interaction between the shock and the ambient plasma. Although a reflection mechanism is not ruled out completely, it is necessary to explain, in the case of the high speed beam, the large amount of momentum gain in the process (Energy is not being conserved in the de HoffmanTeller frame). Moreover, the fact that the ion reflection off a regularly-shaped bow shock fails to account for the beam speed may suggest the occurrence of a local perturbation of the bow shock that will have significant impact on the shock geometry. Incidences of upstream foreshock phenomena as well as small scale solar wind structures on the bow shock have been established by observations; in return, the nature and the efficiency of the shock particle acceleration is affected. For example, large amplitude foreshock ULF waves can significantly inhibit the efficiency of the specular reflection process occurring at quasi-parallel geometries (Meziane et al., 2004a). Various foreshock plasma structures have been reported in the past. Convected back toward the shock, their local impact on the shock surface could be significant. In quasi-parallel regions, convected magnetic pulsations, such SLAMS, play a critical impact in changing the local shock nature. Similar effects are caused by HFAs, characterized by large flow deflections due to the interaction between the solar wind discontinuities with the bow shock (Schwartz et al., 2000). These structures may induce a significant change in the ram pressure, causing a relatively slow outward-inward motion of the shock itself. It results that a local shock surface deformation due to the interaction between the shock and the convecting plasma structures may occur. In the case 
of the high-energy beam, we estimated what would be the scale of such local shock surface deformation that accounts for an ion reflection with conservation of magnetic moment. We have found that at the emission point source, the shock normal is at $\sim 30^{\circ}$ from the normal direction as determined directly from the paraboloid shock surface.

Acknowledgements. Work at UNB has been funded by the Canadian Space Agency.

Guest Editor M. Taylor thanks E. Möbius and another anonymous referee for their help in evaluating this paper.

\section{References}

Balogh, A., Carr, C. M., Acuña, M. H., Dunlop, M. W., Beek, T. J., Brown, P., Fornacon, K.-H., Georgescu, E., Glassmeier, K.H., Harris, J., Musmann, G., Oddy, T., and Schwingenschuh, K.: The Cluster Magnetic Field Investigation: overview of in-flight performance and initial results, Ann. Geophys., 19, 1207-1217, doi:10.5194/angeo-19-1207-2001, 2001.

Bonifazi, C., Moreno, G., and Russell, C. T.: Reflection of the solar wind ions at the Earth's Bow Shock: Energization, J. Geophys. Res., 88, 7853-7859, 1983.

Cairns, I. H., Fairfield, D. H., Anderson, R. R., Carlton, V. E. H., Paularena, K. I., and Lazarus, A. J.: Unusual locations of Earth's bow shock on September 24-25, 1987: Mach number effects, J. Geophys. Res., 100, 47-62, 1995.

Edmiston, J. P., Kennel, C. F., and Eichler, D.: Escape of Heated Ions Upstream of Quasi-Parallel Shocks, Geophys. Res. Lett., 9, 531-534, 1982.

Farris, M. H., Petrinec, S. M., and Russell, C. T.: The thickness of the magnetosheath: constraints on the polytropic index, Geophys. Res. Lett., 18, 1821-1824, 1991.

Gary, S. P., Gosling, J. T., and Forslund, D. W.: The electromagnetic ion beam instability upstream of the Earth's bow shock, J. Geophys. Res., 86, 6691, 1981.

Kis, A., Scholer, M., Klecker, B., Kucharek, H., Lucek, E. A., and Rème, H.: Scattering of field-aligned beam ions upstream of Earth's bow shock, Ann. Geophys., 25, 785-799, doi:10.5194/angeo-25-785-2007, 2007.

Kucharek, H., Möbius, E., Scholer, M., Mouikis, C., Kistler, L. M., Horbury, T., Balogh, A., Rème, H., and Bosqued, J. M.: On the origin of field-aligned beams at the quasi-perpendicular bow shock: multi-spacecraft observations by Cluster, Ann. Geophys., 22, 2301-2308, doi:10.5194/angeo-22-2301-2004, 2004.

Mazelle, C., Le Quéau, D., and Meziane, K.: Nonlinear waveparticle interaction upstream from the Earth's bow shock, Nonlin. Processes Geophys., 7, 185-190, doi:10.5194/npg-7-1852000, 2000.

Mazelle, C., Meziane, K., Le Quéau, D., Wilber, M., Eastwood, J. P., Rème, H., Sauvaud, J.-A., Bosqued, J.-M., Dandouras, I., McCarthy, M., Kistler, L. M., Klecker, B., Korth, A., BavassanoCattaneo, M. B., Lundin, R., and Balogh, A.: Production of Gyrating Ions from Nonlinear Wave-Particle Interaction Upstream from the Earth's Bow Shock: a Case Study from Cluster-CIS, Planet. Space Sci., 51, 785-795, 2003.

Meziane, K., Mazelle, C., Lin, R. P., LeQuéau, D., Larson, D. E., Parks, G. K., and Lepping, R. P.: Three-dimensional observations of gyrating ion distributions far upstream from the Earth's bow shock and their associated with low-frequency waves, J. Geophys. Res., 106, 5731-5742, 2001.

Meziane, K., Mazelle, C., Wilber, M., LeQuéau, D., Eastwood, J. P., Rème, H., Dandouras, I., Sauvaud, J. A., Bosqued, J. M., Parks, G. K., Kistler, L. M., McCarthy, M., Klecker, B., Korth, A., Bavassano-Cattaneo, M.-B., Lundin, R., and Balogh, A.: Bow shock specularly reflected ions in the presence of low-frequency electromagnetic waves: a case study, Ann. Geophys., 22, 23252335, doi:10.5194/angeo-22-2325-2004, 2004a.

Meziane, K., Wilber, M., Mazelle, C., LeQuéau, D., Kucharek, H., Lucek, E., Sauvaud, J. A., Hamza, A. M., Rème, H., Bosqued, J. M., Dandouras, I., Parks, G. K., McCarthy, M., Klecker, B., Korth, A., Bavassano-Cattaneo, M.-B., and Lundin, R.: Simultaneous Observations of Field-Aligned Beams and Gyrating Ions in the Terrestrial Foreshock, J. Geophys. Res., 109, A05107, doi:10.1029/2003JA010374, 2004b.

Meziane, K., Wilber, M., Mazelle, C., Parks, G. K., and Hamza, A. M.: A review of field-aligned beams observed upstream of the bow shock, in: The Physics of Collisionless Shocks, 4th Annual IGPP International Astrophysics Conference, edited by: Li, G., Zank, G. P., and Russell, Christopher, T., vol. 781, pp. 116-122, American Institute of Physics, 2005.

Meziane, K., Wilber, M., Hamza, A. M., Mazelle, C., Parks, G. K., Rème, H., and Lucek, E.: Evidence For a High Energy Tail Associated with Foreshock Field-Aligned Beams, J. Geophys. Res., 112, A01101, doi:10.1029/2006JA011751, 2007.

Meziane, K., Hamza, A. M., Wilber, M., Lee, A. M., Mazelle, C., Lucek, E. A., Tohru, H., and Markowitch, A.: Effect of Shock Normal Orientation Fluctuations On Field-Aligned Beam Distributions, in: The Cluster Active Archive: Studying the Earth's Space Plasma Environment, edited by: Lasco, H., Taylor, M., and Escoubet, P., pp. 349-362, Springer Netherlands, doi:10.1007/978-90-481-3499-1_23, 2010.

Möbius, E., Kucharek, H., Mouikis, C., Georgescu, E., Kistler, L. M., Popecki, M. A., Scholer, M., Bosqued, J. M., Rme, H., Carlson, C. W., Klecker, B., Korth, A., Parks, G. K., Sauvaud, J. C., Balsiger, H., Bavassano-Cattaneo, M.-B., Dandouras, I., DiLellis, A. M., Eliasson, L., Formisano, V., Horbury, T., Lennartsson, W., Lundin, R., McCarthy, M., McFadden, J. P., and Paschmann, G.: Observations of the spatial and temporal structure of field-aligned beam and gyrating ring distributions at the quasi-perpendicular bow shock with Cluster CIS, Ann. Geophys., 19, 1411-1420, doi:10.5194/angeo-19-1411-2001, 2001.

Oka, M., Terasawa, T., Saito, Y., and Mukai, T.: Field-aligned beam observations at the quasi-perpendicular bow shock: Generation and shock angle dependence, J. Geophys. Res., 110, 5631-5649, 2005.

Paschmann, G., Sckopke, N., Asbridge, J. R., Bame, S. J., and Gosling, J. T.: Energization of solar wind ions by reflection from the Earth's bow shock, J. Geophys. Res., 85, 4689-4693, 1980.

Rème, H., Aoustin, C., Bosqued, J. M., Dandouras, I., Lavraud, B., Sauvaud, J. A., Barthe, A., Bouyssou, J., Camus, Th., CoeurJoly, O., Cros, A., Cuvilo, J., Ducay, F., Garbarowitz, Y., Medale, J. L., Penou, E., Perrier, H., Romefort, D., Rouzaud, J., Vallat, C., Alcaydé, D., Jacquey, C., Mazelle, C., d’Uston, C., Mbius, E., Kistler, L. M., Crocker, K., Granoff, M., Mouikis, C., Popecki, M., Vosbury, M., Klecker, B., Hovestadt, D., Kucharek, H., Kuenneth, E., Paschmann, G., Scholer, M., Sckopke, N., Seidenschwang, E., Carlson, C. W., Curtis, D. W., Ingraham, C., Lin, R. 
P., McFadden, J. P., Parks, G. K., Phan, T., Formisano, V., Amata, E., Bavassano-Cattaneo, M. B., Baldetti, P., Bruno, R., Chionchio, G., Di Lellis, A., Marcucci, M. F., Pallocchia, G., Korth, A., Daly, P. W., Graeve, B., Rosenbauer, H., Vasyliunas, V., McCarthy, M., Wilber, M., Eliasson, L., Lundin, R., Olsen, S., Shelley, E. G., Fuselier, S., Ghielmetti, A. G., Lennartsson, W., Escoubet, C. P., Balsiger, H., Friedel, R., Cao, J.-B., Kovrazhkin, R. A., Papamastorakis, I., Pellat, R., Scudder, J., and Sonnerup, B.: First multispacecraft ion measurements in and near the Earth's magnetosphere with the identical Cluster ion spectrometry (CIS) experiment, Ann. Geophys., 19, 1303-1354, doi:10.5194/angeo19-1303-2001, 2001.

Schwartz, S. J., Thomsen, M. F., and Gosling, J. T.: Ions upstream of the Earth's bow shock: a theoretical comparison of alternative source populations, J. Geophys. Res., 88, 2039-2047, 1983.
Schwartz, S. J., Paschmann, G., Sckopke, N., Bauer, T. M., Dunlop, M., Fazakerley, A. N., and Thomsen, M. F.: Conditions for the formation of hot flow anomalies at Earth's bow shock, J. Geophys. Res., 105, 12639-12650, doi:10.1029/1999JA000320, 2000.

Slavin, J. A. and Holzer, R. E.: Solar wind flow about terrestrial planets: 1. Modeling bow shock position and shape, J. Geophys. Res., 86, 11401-11418, 1981.

Sonnerup, B. U. and Scheible, M.: Analysis Methods for MultiSpacecraft Data, chap. Minimum and Maximum Variance Analysis, pp. 185-220, ISSI Scientific Report, ESA Publications Division, Keplerlaan 1, 2200 AG Noordwijk, The Netherlands, 1998.

Thomsen, M. F., Schwartz, S. J., and Gosling, J. T.: Observational evidence on the origin ions upstream of the Earth's bow shock, J. Geophys. Res., 88, 7843-7852, 1983. 